\title{
Pronóstico financiero: métodos rápidos de estimación del fondo de maniobra o capital de trabajo estructural - Caso de una empresa comercial
}

\author{
José Porlles Loarte* \\ Carlos Quispe Atúncar** \\ Gilberto Salas Colottar ***
}

\begin{abstract}
RESUMEN
El crecimiento de la economía del país y por tanto del mercado en todas las actividades empresariales está posibilitando la agresiva expansión de sus ventas. Pero este crecimiento requiere liquidez incremental como capital de trabajo. Los negocios corren el riesgo de limitar su crecimiento si la liquidez que necesitan para asegurarlo excede de la propia generación de fondos. Por tanto, requieren de pronósticos financieros que les permita visualizar los requerimientos de fondos incrementales con la debida anticipación, y conocer si cuentan con los fondos propios generados internamente o gestionar lo necesario en la banca. En este artículo se presenta la metodología e interpretación de resultados en el uso de dos modelos para una estimación rápida de las necesidades de fondos, vale decir, el fondo de maniobra adicional estructural para sostener el incremento de las ventas.
\end{abstract}

Palabras clave: pronóstico financiero, fondo de maniobra, capital de trabajo, liquidez, crecimiento

FINANCIAL FORECAST: FAST METHODS OF ESTIMATION OF WORKING CAPITAL STRUCTURAL - CASE COMERCial ENTERPRISE

\section{ABSTRACT}

The growth of the country's economy and therefore the market in all business activities is enabling the aggressive expansion of its sales. But this growth requires incremental liquidity as working capital. The business run the risk of limiting their growth if the liquidity needed to secure it exceeds the own generation of funds. Therefore require financial forecasts that they can visualize the incremental funds requirements well in advance, and know if they have with their own funds generated internally or manage what is necessary in the banking. In this article presents the methodology and interpretation of results in the use of two models for a quick estimate of the needs of funds, namely, the fund additional maneuver structural to sustain the increase in sales.

Keywords: financial forecast, fund of maneuver, working capital, liquidity, grow

\section{INTRODUCCIÓN}

Es preocupación empresarial la determinación de la necesidad de fondos para sostener las operaciones comerciales. Cuando una empresa recién inicia sus actividades comerciales requiere un capital base para asegurar las operaciones en el largo plazo; este capital circulante se denomina Fondo de Maniobra o Capital de Trabajo Estructural, monto que debe ser contemplado dentro del plan de inversión y financiamiento. Una empresa en marcha que no altera su nivel de actividad, no requerirá de capital circulante adicional.

El problema surge cuando la empresa en marcha pronostica crecimientos de su actividad, que definitivamente si no cuenta con la liquidez adicional correspondiente no podrá afrontarla.

Entonces, en primer lugar, necesita conocer la cuantía de fondos adicionales para sostener un determinado aumento de las ventas. En segundo lugar, deberá determinar si los fondos generados en su ciclo operativo anterior ayudarán a autofinanciar el ciclo de operación siguiente o si bien tendrá que recurrir a financiamiento externo vía crédito bancario o aumento de capital accionario adicional.

El mayor o menor requerimiento de fondos por una empresa, está íntimamente vinculado con la administración del Capital de Trabajo o Fondo de Maniobra, así como con la financiación de los activos circulantes. Si la empresa es consciente que está generando fondos, entonces es posible que sus requerimientos sea financiada por autogeneración de efectivo; si no es suficiente, lo restante debe ser financiada con fuentes externas.

El propósito del presente trabajo contribuye a potenciar y esclarecer las metodologías que pueden ser útiles para los directivos o analistas económicos de empresas en marcha, en la determinación de los fondos requeridos como capital circulante o la variación del Fondo de Maniobra para sostener ventas incrementales futuras en una empresa comercial.

Para dicho efecto se ha considerado el aporte de trabajos de investigación anteriores y vinculantes de autores que se

\footnotetext{
Magíster en Administración, Profesor del Departamento de Análisis y Diseño de Procesos. UNMSM. E-mail: joseporlles@yahoo.com

** Ingeniero Industrial, Profesor del Departamento de Ingeniería de Sistemas e Informática de la UNMSM.

** Ingeniero Químico. Profesor del Departamento de Operaciones Unitarias de la UNMSM. E-mail: gsalas19@yahoo.es, cquispea@unmsm.edu.pe
} 
señalan a continuación: Ramírez (2011), Herrera (2007), Rizzo (2007) y, Selpa y Espinosa (2009). En algunos casos se ha adecuado su tratamiento para una mejor comprensión e interpretación por el usuario.

\section{INFORMACIÓN BASE NECESARIA}

Para efectos de análisis se requiere como mínimo:

1. Contar con el estado de resultados (EPG) que refleja la gestión operativa.

2. El EPG representativo se debe traducir en un EPG en términos de caja (excluyendo los cargos no desembolsables), con una estructura porcentual adecuada para el análisis.

3. Contar con el balance general que representa la gestión financiera en un periodo determinado. De este cuadro se deben obtener los parámetros vinculados a los periodos: cuentas por cobrar (clientes), existencias (inventarios) y cuentas por pagar (proveedores).

\subsection{Ilustración de una empresa comercial}

Para comprender el análisis e interpretar los resultados, en este trabajo se toma en cuenta la información para una empresa comercial cuyos estados financieros se muestran en el Anexo 1; presentándose además el análisis financiero resultado de las políticas financieras y operativas de la compañía, tal como se ilustra en el Anexo 2. Se trata de una empresa ubicada cerca al río Itaya adyacente a la ciudad de Loreto en el oriente peruano. Comercializa al por mayor triplay (madera terciada), molduras y marcos de madera para puertas y ventanas.

A pesar que ha venido reteniendo las utilidades en el negocio, la compañía había experimentado cierta falta de efectivo, por lo que se había visto obligada a solicitar un préstamo de $\$ 48,000$ en el primer trimestre del año bajo análisis, el mismo que le fue otorgado. Sin embargo, las perspectivas de mercado configuraban la necesidad de solicitar un préstamo adicional para sostener su crecimiento de ventas, lo que implicaba estimar estas necesidades de fondos. Tal situación permitirá la aplicación de los modelos que se proponen más adelante.

La gerencia presentó su requerimiento adicional a un banco local en Lima por $\$ 80000$, estando en pleno proceso de evaluación. Investigaciones previas indican que las ventas pueden fluctuar entre $\$ 1,400000$ y $\$ 1,650000$ en el año en curso.

\section{MODELO 1: ROTACIÓN DE EFECTIVO Y POR- CENTAJE DE VARIACIÓN DE VENTAS}

Este modelo centra su atención en las variaciones de las partidas: caja, clientes, existencias y proveedores.

La metodología es la siguiente:

1. Tomando en cuenta la información del estado de resultados (EPG) consignada en la figura 1 para las dos alternativas de posibles ventas, se estima: las ventas incrementales, los costos de ventas adicionales, el incremento de compras adicionales y la generación de utilidades como se ilustra en el cuadro 1.

Cuadro 1. Análisis de alternativas

\begin{tabular}{|l|r|r|}
\hline \multicolumn{1}{|c|}{ Partidas en miles de \$ } & \multicolumn{1}{|c|}{ Alt. 1 } & \multicolumn{1}{|c|}{ Alt. 2 } \\
\hline Ventas previstas $(\mathrm{V})$ & 1,400 & 1,650 \\
\hline $\begin{array}{l}\Delta \mathrm{V} \text { : ventas adicionales (en relación a } \\
\text { la venta del año 2: } 1,179 \text { miles de } \$)\end{array}$ & 221 & 471 \\
\hline $\begin{array}{l}\Delta \mathrm{CV}: \text { costo de ventas adicionales } \\
(88 \% / \Delta \mathrm{V} \text {, valor de la figura 1) }\end{array}$ & 194 & 414 \\
\hline $\begin{array}{l}\text { Utilidad neta (4\%/V, valor de la } \\
\text { Figura 1) }\end{array}$ & 56 & 66 \\
\hline $\begin{array}{l}\Delta \text { Compras: compras adicionales } \\
(93 \% / \Delta \mathrm{V}, \text { valor en el Anexo 2) }\end{array}$ & 205 & 438 \\
\hline
\end{tabular}

Fuente: Elaboración propia.

2. Ajustar, de ser necesario, las condiciones financieras de operación. En el caso se tiene:

- Caja mínima: el promedio para cinco días de venta (parámetro aceptable de manera general y cuyo cálculo se anota al pie del Anexo 1).

- Cuentas por cobrar: el promedio de 32 días se mantiene según tendencia y sirve para calcular el aumento en cuentas por cobrar usando el ratio financiero del Anexo 2.

- Existencias: el promedio de 60 días se mantiene según tendencia y sirve para calcular el aumento en existencias usando el ratio financiero del Anexo 2.

- Cuentas por pagar: el promedio de 48 días, se reduce a 30 días y sirve para calcular la disminución de cuentas por pagar usando el ratio financiero del Anexo 2. 
3. Los cálculos previos permiten computar la necesidad de fondos adicionales, sobre la base de cálculo de las variaciones incrementales de los componentes de la cuenta: clientesexistencias-proveedores, descontando al monto anterior los fondos generados internamente, resultando los fondos que deben ser financiados vía financiamiento externo, como se ilustra en el Cuadro 2.

Cuadro 2. Necesidad de financiamiento externo

\begin{tabular}{|l|r|r|}
\hline \multicolumn{3}{|c|}{ Necesidad de fondos en miles de \$ para cubrir: } \\
\hline Partida & Alt. 1 & \multicolumn{1}{|c|}{ Alt. 2 } \\
\hline $\begin{array}{l}\text { Aumento de caja mínima (cálculos } \\
\text { al pie del Anexo 1) }\end{array}$ & 3 & 7 \\
\hline $\begin{array}{l}\text { Aumento cuentas por cobrar }(\Delta \mathrm{C} . \\
\text { c = } \Delta \text { V (32 días)/(360 días) }\end{array}$ & 20 & 42 \\
\hline $\begin{array}{l}\text { Aumento existencias ( } \Delta \text { C.c }=\Delta C V \\
\text { (60 días)/(360 días) }\end{array}$ & 32 & 69 \\
\hline $\begin{array}{l}\text { Disminución cuentas por pagar (en } \\
\text { cálculos auxiliares del anexo 2) }\end{array}$ & 63 & 40 \\
\hline $\begin{array}{l}\text { Necesidad de Fondos } \\
\text { adicionales: }\end{array}$ & 118 & 158 \\
\hline $\begin{array}{l}\text { Menos fondos generados como } \\
\text { Utilidad neta }\end{array}$ & 56 & 66 \\
\hline Necesidad de fondos externos & 62 & 92 \\
\hline
\end{tabular}

Fuente: Elaboración propia

Debe apreciarse en este caso que la partida cuenta por pagar no es una fuente de fondos; al rebajar el periodo de pago se convierte en una partida que requiere fondos para financiar las mayores compras.

Los resultados indican que en ambas alternativas la empresa requeriría apoyo financiero externo para sostener su ampliación de ventas. Sin embargo, como ha recibido un crédito de $\$ 48,000$ en el primer trimestre del año bajo evaluación, entonces el crédito adicional debería se por un monto de $\$ 14,000$ para el primer escenario y de $\$ 44,000$ para la alternativa 2 .

Una referencia interesante sobre esta metodología puede consultarse en la obra de Lira (2012).

\section{MODELO 2: POTENCIAL DE CRECIMIENTO Y CICLO OPERATIVO DE CAJA (COC)}

Este modelo es mucho más elaborado y hace uso de dos conceptos íntimamente vinculados: el ciclo operativo de caja (COC) y la inversión inmovilizada en LIQUIDEZ para generar 1 \$ en ventas.

\subsection{Conceptualización del COC}

Es de conocimiento generalizado que las empresas registran un ciclo de operación que está en función del comportamiento de variables operativas y de decisiones financieras: compra de materiales, periodos de existencia de los mismos, periodo de procesamiento de dichos materiales en productos finales y periodo de almacenamiento hasta su venta y facturación; y finalmente la modalidad de las ventas: si es al crédito o al contado.

Ross et al. (2000) hacen una diferenciación de lo que es el ciclo operativo a corto plazo y ciclo de flujo de efectivo. Anotan que el ciclo operativo es el periodo que transcurre desde la llegada de las mercancías al inventario hasta la recepción del efectivo a partir de las cuentas por cobrar, incluyendo el lapso comprendido desde la colocación de la orden hasta la llegada de la mercancía. El ciclo de efectivo empieza cuando se paga efectivo por los materiales y termina cuando se recibe efectivo en contraparte de las cuentas por cobrar.

La longitud del ciclo operativo es igual a la suma de las longitudes de los periodos del inventario y las cuentas por cobrar. El periodo del inventario es el lapso que se requiere para ordenar, producir y vender un producto. El periodo de las cuentas por cobrar se traduce en el tiempo que se requiere para cobrar los ingresos en efectivo.

Entonces, Ross (2000) concibe que el ciclo de efectivo es el tiempo que transcurre entre los desembolsos de efectivo y la cobranza, es decir es equivalente al ciclo operativo menos el periodo de las cuentas por pagar.

Churchill y Mullins (2001) conciben un solo ciclo denominado: el ciclo operativo de caja (COC); anotando "la duración del ciclo operativo de caja es la del componente de la liquidez requerida para producir una unidad de venta que durante más tiempo esté inmovilizada". Significa entonces que el ciclo operativo no solo está conformado por las inversiones en efectivo inmovilizadas en el tiempo de las partidas cliente, existencia y proveedores, sino que debe incluirse el lapso de tiempo de los gastos de explotación y de otros gastos que se pueden distribuir a lo largo de periodos diferentes como puede ser el caso de gastos anticipados 
en investigación de mercado o de anuncios publicitarios en general. El ciclo operativo de caja es equivalente al más largo de todos estos ciclos.

Para una comprensión cabal del uso de dicha técnica, se va a utilizar la información de la ilustración que se viene analizando. En la Figura 1 se representa el COC para la empresa comercial objeto de evaluación, la estructura del EPG y las condiciones de política financiera.

La gran ventaja de este modelo es que permite responder a dos interrogantes que todo buen empresario desea conocer: a) determinar las necesidades de fondos para sostener un crecimiento de ventas $\mathrm{y}, \mathrm{b})$ ¿a qué ritmo se puede crecer sostenidamente con la capacidad de autogeneración, sin necesidad de recurrir a fuentes de financiamiento externo?

La metodología de trabajo es la siguiente:

1. Determinar el lapso de inmovilización de la inversión en "existencias" antes de venderlos, vale decir hasta cuando se expide la factura de ventas; en este caso son 60 días.

2. Establecer el periodo de tiempo de inversión inmovilizada hasta hacer efectiva la cobranza; en este caso de 32 días.

3. Determinar el ciclo operativo de caja (COC) que en el caso es de 92 días.

4. Determinar si se cuenta con crédito de proveedores. En el ejemplo se cuenta con 30 días, por lo que en realidad el efectivo en forma de "existencias" solo está inmovilizado durante 62 días.

5. Determinar la inversión inmovilizada del capital circulante dentro del COC en términos de días, que abarca 62 días. En la literatura especializada a este periodo se le conoce como “aprovisionamiento", es el inmovilizado de la mercadería desde que entra a la empresa y se efectiviza la cobranza de dicha mercadería.

Figura 1. Información base (COC de la empresa comercial = 92 días)

(Tomando datos promedio del Anexo 2)

\begin{tabular}{|l|c|}
\hline \multicolumn{2}{|l|}{ Estado de resultados (EPG) } \\
\hline Ventas & $100 \%$ \\
Costo de ventas & $88 \%$ \\
(Aprovisionamiento de materiales) & \\
Gastos de explotación & $\underline{8 \%}$ \\
Utilidad & $4 \%$ \\
\hline
\end{tabular}

\begin{tabular}{|l|c|}
\hline \multicolumn{2}{|l|}{ Gestión Financiera (del Balance General) } \\
\hline$\underline{\text { Cuenta }}$ & $\underline{\text { Días }}$ \\
Existencias & 60 \\
Cuentas por cobrar & $\underline{32}$ \\
COC & 92 \\
Cuentas por pagar & 30 \\
Aprovisionamiento & 62 \\
Gastos de explotación (Operativos) & 46 \\
\hline
\end{tabular}

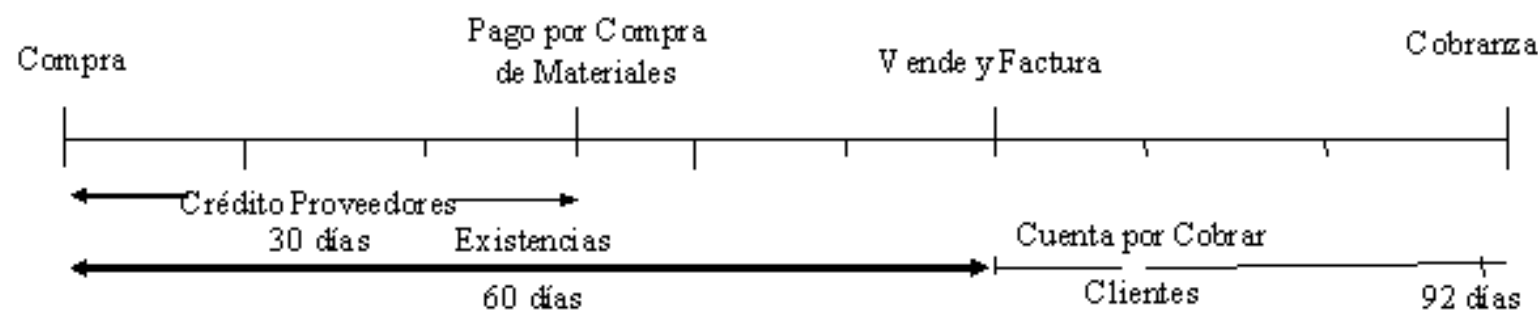


6. Determinar el tiempo que se requiere de efectivo para cubrir los gastos de explotación (planilla de ejecutivos y trabajadores, gastos administrativos, gastos de ventas y otros conexos) que en promedio se incurren diariamente. Se asume que este rubro se gasta uniformemente a lo largo de los 92 días del COC. En realidad, en el promedio la inversión inmovilizada en esta cuenta corresponde a la mitad del periodo del COC, vale decir 46 días en este caso.

7. Determinar si el total de la utilidad prevista por cada una unidad de venta se retiene en la empresa para reinversiones o aumento del capital circulante o si parte será distribuido como dividendo o pago a cuenta de utilidades. En este caso se asume retención de utilidades y como se excluye la depreciación en el EPG, la generación de fondos está dado por la utilidad.

En resumen para el caso de empresas comerciales se tiene inmovilización de efectivo en dos grandes rubros:

1. Inversión en costo de ventas como aprovisionamiento: que está vinculada a las cuentas: clientes, existencias y proveedores: se inmoviliza efectivo como cuentas por cobrar y existencias, pero descontando cuentas por pagar.

2. Inversión en gastos de explotación: que se estima gasto uniforme a lo largo del COC. No es un gasto tipo stock congelado desde el inicio de operaciones, se va recuperando según se concreta la cobranza.

Luego la inversión inmovilizada de fondos para generar \$ 1 de venta, se consigna en el Cuadro 3.

\subsection{Análisis del crecimiento sostenido}

Se refiere a la potencialidad de la empresa para autosostener su crecimiento futuro y el cálculo se ilustra en el Cuadro 4.

La tasa de crecimiento anual de $24.6 \%$ es la tasa máxima de crecimiento autofinanciable, permitiendo pronosticar las necesidades de fondos de la empresa. Es autofinanciable para un nivel próximo de ventas de 1469 miles de dólares. Significa entonces lo siguiente: si la empresa proyecta un crecimiento de ventas anuales en un porcentaje inferior a dicha tasa anual, la compañía está generando más liquidez con sus ventas de la que su crecimiento requiere. Pero si la empresa proyecta crecer sus ventas en un porcentaje superior a la referida tasa autofinanciable, definitivamente requiere gestionar fondos de fuentes externas.
Cuadro 3. Inversión inmovilizada para generar \$ 1 de venta

Base: para generar $1 \$$ de ventas

a) Inversión en existencias en términos de costo de ventas (dato del EPG en la Figura 1)

$\$ 0,88$

$>$ Efectivo inmovilizado en días (como aprovisionamiento: ver Figura 1)

$>$ Efectivo inmovilizado en existencias durante el COC $(\$ 0,88)(62 / 92)$

b) Inversión en gastos de explotación ( $8 \%$ de ventas: ver EPG en la Figura 1; gastos operativos desembolsables)

$>$ Efectivo inmovilizado en días (92/2)

> Efectivo inmovilizado para financiar dichos gastos $(\$ 0,08)(46 / 92)$

Efectivo total invertido para generar $1 \$$ de ventas $(\mathbf{a}+\mathbf{b})$

(Capital Circulante en el COC)

Fuente: Elaboración propia

Cuadro 4. Tasa de crecimiento autofinanciable

\begin{tabular}{|l|c|}
\hline $\begin{array}{l}\text { a) Efectivo invertido en el ciclo precedente } \\
\text { para generar \$1 de venta }\end{array}$ & $\mathbf{\$ 0 , 6 3}$ \\
\hline $\begin{array}{l}\text { b) Beneficios que pueden ser reinvertidos } \\
\text { (Ver EPG en la Figura 1) }\end{array}$ & $\$ 0,04$ \\
\hline $\begin{array}{l}\text { c) Efectivo proyectado para el siguiente } \\
\text { ciclo (autogenerado) (a+b) }\end{array}$ & $\mathbf{\$ 0 , 6 7}$ \\
\hline $\begin{array}{l}\text { Tasa de crecimiento autofinanciable para el } \\
\text { COC próximo (0,67/0,63) }\end{array}$ & $6,3 \%$ \\
\hline $\begin{array}{l}\text { Tasa de crecimiento autofinanciable } \\
\text { anual }(\mathbf{6 , 3} \%)(360 \text { días/92 días del COC) }\end{array}$ & $\mathbf{2 4 , 6 \%}$ \\
\hline
\end{tabular}

Fuente: Elaboración propia. 
Las condiciones imperantes en el mercado, el tipo de tecnología de fabricación y las políticas financieras que implemente la empresa, determinará el potencial de crecimiento autofinanciable en una empresa en marcha, como herramienta para pronosticar la necesidad de fondos para sostener futuros crecimientos en un mercado de competencia.

En el Cuadro 5 se visualiza los resultados finales de necesidad operativa de fondos para las dos alternativas bajo análisis.

Cuadro 5. Necesidad operativa de fondos

\begin{tabular}{|c|c|c|}
\hline Partida & Alt. 1 & Alt. 2 \\
\hline $\begin{array}{l}\text { V: ventas anuales previstas en miles } \\
\text { de } \$\end{array}$ & 1400 & 1650 \\
\hline $\begin{array}{l}\text { Crecimiento de ventas proyectado } \\
\text { en relación a las ventas del año } \\
\text { anterior que fue: } 1179 \text { miles de } \$\end{array}$ & $18,8 \%$ & $40,0 \%$ \\
\hline Utilidad (4\%/N, del EPG en la Figura 1$)$ & 56 & 66 \\
\hline \multicolumn{3}{|c|}{ Necesidad Operativa de Fondos (miles de \$): } \\
\hline $\begin{array}{l}\text { a) Inversión en Capital Circulante } \\
\text { (efectivo inmovilizado) } \\
\checkmark \quad(\$ 0,63)(1400)=882 \\
\checkmark \quad(\$ 0,63)(1650)=1040\end{array}$ & 882 & 1,040 \\
\hline $\begin{array}{l}\text { b) Liquidez generada para auto- } \\
\text { financiar crecimiento en } 24,6 \% \\
\checkmark(\$ 0,63)(1,246)(1179 \text { miles de } \$)\end{array}$ & 925 & 925 \\
\hline c) Financiamiento externo (a-b) & (43) & 115 \\
\hline
\end{tabular}

Fuente: Elaboración propia

En la alternativa 1 los fondos generados internamente cubren a satisfacción el crecimiento proyectado que es menor a $24,6 \%$; pero en la alternativa 2 se necesita fondos del exterior por \$ 115000 , y deduciendo los \$ 48000 ya obtenidos solo requiere apoyo financiero adicional por $\$ 67000$.

En la segunda publicación se evaluará los requerimientos de liquidez para una empresa manufacturera, que requiere mayores consideraciones de análisis.

\section{CONCLUSIONES}

5.1 Para los dos métodos usados en este documento, los resultados resumidos se señalan en el Cuadro 6, registrándose cifras disímiles que pueden conducir a decisiones diferentes, por lo que hay que tener en cuenta los aspectos que se señalan más adelante.

Cuadro 6. Resultados resumidos de ambos modelos

\begin{tabular}{|c|c|c|}
\hline \multicolumn{3}{|c|}{$\begin{array}{l}\text { 1) Modelo de rotación de efectivo y porcentaje de } \\
\text { variación de ventas }\end{array}$} \\
\hline & Alt. 1 & Alt. 2 \\
\hline Necesidad de fondos adicionales & 118 & 158 \\
\hline $\begin{array}{l}\text { Menos fondos generados como } \\
\text { utilidad neta }\end{array}$ & 56 & 56 \\
\hline $\begin{array}{l}\text { Necesidad de financiamiento } \\
\text { externo }\end{array}$ & 62 & 92 \\
\hline \multicolumn{3}{|c|}{ 2) Modelo potencial de crecimiento y COC } \\
\hline & Alt. 1 & Alt. 2 \\
\hline $\begin{array}{l}\text { Inversión en capital circulante } \\
\text { como inmovilizado }\end{array}$ & 882 & 1040 \\
\hline $\begin{array}{l}\text { Menos liquidez generada para } \\
\text { autofinanciar crecimiento }\end{array}$ & 925 & 925 \\
\hline $\begin{array}{l}\text { Necesidad de financiamiento } \\
\text { externo }\end{array}$ & (43) & 115 \\
\hline
\end{tabular}

Fuente: Elaboración propia.

5.2 Si bien ambos modelos en sus cálculos usan las mismas premisas básicas que se asuman en las condiciones de políticas financieras y de operación (EPG), los cuales se reflejan en los periodos de las partidas caja, clientes, existencias y proveedores, la diferencia estriba en los temas siguientes:

a) El modelo rotación de efectivo considera las variaciones de las partidas: caja, clientes, existencias y proveedores de manera aislada y en función del incremento de ventas estimado.

b) El modelo COC introduce el concepto de liquidez inmovilizado en dos aspectos: inversión en las partidas cliente, existencia y proveedores, que en este caso son 62 días y se le conoce bajo la denominación de "aprovisionamiento" y es el inmovilizado de la mercadería desde que entra a la empresa y se efectiviza la cobranza; incluyendo además, la inversión por el lapso de tiempo de los gastos de explotación, que en el caso registra 46 días. 
El concepto de inversión inmovilizada en liquidez para generar 1 \$ en ventas y el potencial de crecimiento para autosostenerse, son conceptos que le dan un carácter dinámico al modelo COC.

\section{RECOMENDACIONES}

6.1. El modelo de rotación de efectivo se puede usar cuando se hace un estudio muy preliminar y se requiere también una estimación muy rápida. Su resultado proporciona una primera idea de la necesidad de fondos para una variación incremental de ventas. Se sugiere no usarlo para formalizar un requerimiento de apoyo financiero externo.

6.2. El modelo COC es útil en un estudio de mayor profundidad que requiere mayor aproximación; su resultado tiende a ser más realista de las necesidades de fondos incrementales para sostener saltos de ventas de tipo estructural, que se hacen permanentes en el largo plazo.

\section{BIBLIOGRAFÍA}

[1] Churchill, N. y Mullins, J. (2001). How Fast Can Your Company Afford to Grow?, Harvard Business Review, May 2001. Tool Kit Reprint 0105K.

[2] Internet: http://www.leaderslab oratory.com/wpcontent/uploads/2013/02/HBR-How-Fast-CanYour-Company-Afford-to-Grow.pdf

Anexo 1. Estados Financieros Estado de Resultados (EPG en miles de \$)

\begin{tabular}{|l|r|r|r|}
\hline \multicolumn{1}{|c|}{ Partida } & Año 1 & Año 2 & Año 3* \\
\hline Ventas netas & 880 & 1179 & 310 \\
\hline Costo art. vendidos & & & \\
\hline Inventario al 1 de enero & 97 & 141 & 180 \\
\hline Compras & 846 & 1069 & 336 \\
\hline Subtotal & 943 & 1210 & 516 \\
\hline Saldo inventario & 141 & 180 & 244 \\
\hline Costo de ventas & 802 & 1030 & 272 \\
\hline Utilidad bruta & 78 & 149 & 38 \\
\hline Gastos de operación & 48 & 73 & 20 \\
\hline Utilidad de operación & 30 & 76 & 18 \\
\hline Más: descuentos obtenidos & 0,5 & 0,5 & 0,6 \\
\hline & 35 & 81 & 19 \\
\hline $\begin{array}{l}\text { Menos: descuentos } \\
\text { otorgados }\end{array}$ & 18 & 28 & 08 \\
\hline Utilidad neta & 17 & 53 & 11 \\
\hline
\end{tabular}

*Corresponde al primer trimestre del Año 3.
[3] Herrera, A. (Julio 2007). Internet: http://www. monografias.com/trabajos70/argumentacionteorica-capital-trabajo/argumentacion-teoricacapital-trabajo2.shtml

[4] Lira Briceño, Paul (2012). Métodos para Estimar el Capital de Trabajo. Parte 1 al 3. Blogs Gestión, publicados en Junio 2012. Internet:http:// blogs.gestion.pe/deregresoalobasico/2011/01/ metodos-para-estimar-el-capita.html

[5] Ramírez, G. (2011). ¿Cómo calcular el capital de trabajo que necesito? Junio 2011.

[6] Internet: www.cityen.cl/tag/capital-de-trabajo.html

[7] Rizzo, M. (2007). El capital de trabajo neto y el valor en las empresas. Revista EAN N. ${ }^{\circ} 11$, Octubre 2007, pp. 103-122.

[8] Ross, S., Westerfield, R. y Jaffe, J. (2000). Finanzas Corporativas, McGraw Hill, 5. ${ }^{a}$ ed, 2000. Capítulo 27, pp. 822-840.

[9] Selpa, A. y Espinosa, D. (2009). La Gestión del Capital de Trabajo como Proceso de la Gestión Financiera Operativa. Universidad de Matanzas Camilo Cienfuegos, Departamento de Profesores de Contabilidad y Finanzas, Cuba. Internet:http:// www.elcriterio.com/revista/ajoica/contenidos_4/ ambar_selpa_y_daisy_espinosa.pdf

\section{Balance General (Miles de \$)}

\begin{tabular}{|l|r|r|r|}
\hline \multicolumn{1}{|c|}{ Activo } & Año 1 & Año 2 & Año 3 \\
\hline Caja & 1 & 4 & 1 \\
\hline Cuenta por cobrar & 89 & 110 & 129 \\
\hline Inventario & 142 & 180 & 244 \\
\hline Activo corriente & $\mathbf{2 3 1}$ & $\mathbf{2 9 3}$ & $\mathbf{3 7 4}$ \\
\hline Activo fijo neto & 8 & 11 & 10 \\
\hline Cargas diferidas & & & 3 \\
\hline Activo Total & $\mathbf{2 3 9}$ & $\mathbf{3 0 4}$ & $\mathbf{3 8 7}$ \\
\hline Pasivo & & & \\
\hline Doc. por pagar BankSelva & & & 48 \\
\hline Doc. por pagar empleados & & & 5 \\
\hline Efectos por pagar & & & 66 \\
\hline Cuentas por pagar & 137 & 174 & 138 \\
\hline Gastos acumulados & 3 & 7 & 1 \\
\hline Pasivo corriente & $\mathbf{1 4 0}$ & $\mathbf{1 8 1}$ & $\mathbf{2 5 8}$ \\
\hline Capital neto & 99 & 123 & 129 \\
\hline Pasivo Total & 239 & 304 & 387 \\
\hline
\end{tabular}


Anexo 2. Análisis Financiero

\section{Cálculo de Ratios Financieros}

\begin{tabular}{|c|c|c|c|}
\hline Partida & Año 1 & Año 2 & Año 3 \\
\hline \multicolumn{4}{|c|}{ A) Cuenta por cobrar (C. c) } \\
\hline \multicolumn{4}{|c|}{ Rot. C. $\mathrm{c}=$ Ventas / C. c promedio } \\
\hline C. c al inicio $\$$ & 57 & 89 & 110 \\
\hline C. c al final $\$$ & 89 & 110 & 128 \\
\hline C. c promedio $\$$ & 73 & 10 & 119 \\
\hline Ventas \$ & 880 & 1179 & 310 \\
\hline Rotación C. c & 12,1 & 11,8 & 2,6 \\
\hline N. ${ }^{\circ}$ de días:360/Rot & 30 & 31 & 35 \\
\hline \multicolumn{4}{|l|}{ B) Existencias } \\
\hline \multicolumn{4}{|c|}{ Rot. Existencias = Costo de ventas / Exist. Promedio } \\
\hline Exist. al inicio \$ & 97 & 142 & 180 \\
\hline Exist. al final $\$$ & 142 & 180 & 244 \\
\hline Exist. promedio $\$$ & 120 & 161 & 212 \\
\hline Costo de ventas & 802 & 1030 & 272 \\
\hline Rotación Exist. & 6,7 & 6,4 & 1,3 \\
\hline N. ${ }^{\circ}$ de días: $360 /$ Rot & 54 & 56 & 69 \\
\hline \multicolumn{4}{|c|}{ C) Cuenta por pagar (C. p) } \\
\hline \multicolumn{4}{|c|}{ Rot. C. $p=$ compras / C. $p$ promedio } \\
\hline C. $p$ al inicio $\$$ & 58 & 137 & 174 \\
\hline C. $p$ al final $\$$ & 137 & 174 & 204 \\
\hline C. p promedio $\$$ & 98 & 156 & 189 \\
\hline Compras \$ & 846 & 1069 & 336 \\
\hline Rotación C. p & 8,6 & 6,9 & 1,8 \\
\hline N. ${ }^{\circ}$ de días: $360 /$ Rot & 42 & 52 & 50 \\
\hline
\end{tabular}

Estructura Porcentual (\%) del EPG

\begin{tabular}{|l|r|r|r|}
\hline Partida & Año 1 & Año 2 & Año 3 \\
\hline Ventas & 100 & 100 & 100 \\
\hline Compras & 96 & 91 & 108 \\
\hline Costo de art. vendidos & 91 & 87 & 88 \\
\hline Gastos de operación & 6 & 6 & 6 \\
\hline Descuentos otorgado neto & 2 & 2 & 2 \\
\hline Utilidad neta & $\mathbf{2}$ & $\mathbf{5}$ & $\mathbf{4}$ \\
\hline
\end{tabular}

\section{Cálculos auxiliares de C. p final:}

\section{Alternativa 1}

Compras: $(93 \% * 1400$ miles $\$)=1302$ miles $\$$ sumado al aumento de inventario de 32 miles $\$$ se tiene compras finales de 1334 miles \$

\section{Cálculo de caja mínima Incremental:}

Alt. 1: 221 miles $\$ / 360$ días: $0.614 \$$ /día

Para 5 días: $(0.614 * 5$ días $)=3$ miles $\$$

Alt. 2: 471 miles $\$ / 360$ días: $1,3 \$$ día

Para 5 días: $(1,3 * 5$ días $)=7$ miles $\$$

$30=($ C. $p * 360) / 1334$

C. $p$ final $=111$ miles $\$$

C. $p$ inicial $=174$ miles $\$($ fin del Año 2)

Disminución C. $p=63$ miles $\$$ 